\title{
Cost-Effectiveness Analysis of Prognostic Gene Expression Signature-Based Stratification of Early Breast Cancer Patients
}

\author{
Patricia R. Blank • Martin Filipits • Peter Dubsky • Florian Gutzwiller • \\ Michael P. Lux $\cdot$ Jan C. Brase $\cdot$ Karsten E. Weber - Margaretha Rudas • \\ Richard Greil - Sibylle Loibl - Thomas D. Szucs • Ralf Kronenwett • \\ Matthias Schwenkglenks • Michael Gnant
}

Published online: 18 November 2014

(C) The Author(s) 2014. This article is published with open access at Springerlink.com

\begin{abstract}
Background The individual risk of recurrence in hormone receptor-positive primary breast cancer patients determines whether adjuvant endocrine therapy should be combined with chemotherapy. Clinicopathological parameters and molecular tests such as EndoPredict ${ }^{\mathbb{B}}$ (EPclin) can support decision making in patients with estrogen receptor-positive, human epidermal growth factor receptor 2 (HER2)negative cancer.

Objective Using a life-long Markov state transition model, we determined the health economic impact and incremental cost effectiveness of EPclin-based risk stratification in combination with clinical guidelines [GermanS3, National Comprehensive Cancer Center Network (NCCN), and St. Gallen] to decide on chemotherapy use.
\end{abstract}

On behalf of the Responsify consortium: Sibylle Loibl.

M. Schwenkglenks and M. Gnant shared senior authorship.

Electronic supplementary material The online version of this article (doi:10.1007/s40273-014-0227-x) contains supplementary material, which is available to authorized users.

P. R. Blank $(\bowtie) \cdot$ F. Gutzwiller · T. D. Szucs ·

M. Schwenkglenks

Institute of Pharmaceutical Medicine (ECPM), University of

Basel, Klingelbergstrasse 61, 4056 Basel, Switzerland

e-mail: patricia.blank@unibas.ch

M. Filipits

Department of Medicine I, Medical University of Vienna,

Vienna, Austria

P. Dubsky · M. Gnant

Department of Surgery, Medical University of Vienna, Vienna,

Austria

M. P. Lux

Universitäts-Brustzentrum Franken, Frauenklinik,

Universitätsklinikum Erlangen, Erlangen, Germany
Methods Information on overall and metastasis-free survival came from Austrian Breast \& Colorectal Cancer Study Group clinical trials $6 / 8(n=1,619)$ and published literature. Effectiveness was assessed as quality-adjusted life-years (QALYs). Costs (2010) were assessed from a German third-party payer perspective.

Results Lifetime costs per patient ranged from $€ 28,268$ (St.Gallen and EPclin) to $€ 33,756$ (NCCN). Due to an imperfect prognostic value and differences in chemotherapy use, strategies achieved between 13.165 QALYs (NCCN) and 13.173 QALYs (EPclin alone) per patient. Using German-S3 as reference, three strategies showed dominant results (St. Gallen and EPclin, German-S3 and EPclin, EPclin alone). Compared to German-S3, the addition of EPclin saved $€ 3,388$ and gained 0.002 QALYs per patient. Combining guidelines with EPclin remained preferable in sensitivity analysis.

Conclusion Our study suggests that molecular markers can be sensibly combined with clinical guidelines to determine the risk profile of adjuvant breast cancer

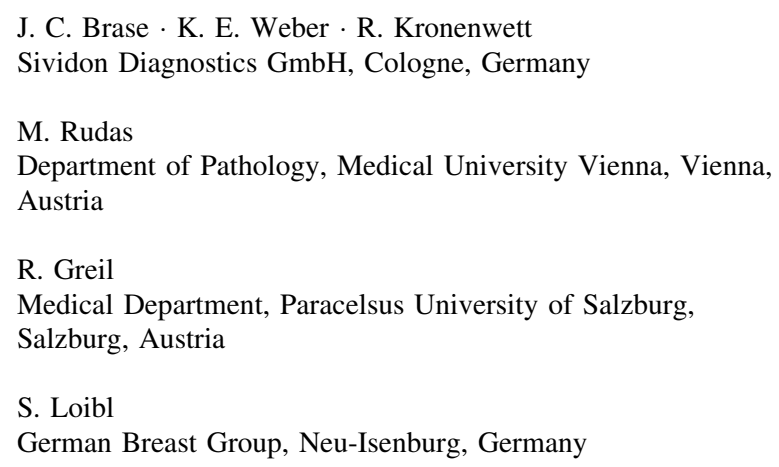


patients. Compared with the current German best practice (German-S3), combinations of EPclin with the St. Gallen, German-S3 or NCCN guideline and EPclin alone were dominant from the perspective of the German healthcare system.

\section{Key Points for Decision Makers}

In current practice, clinical and pathological factors, but also molecular tests, are used to assess the individual risk of recurrence among early estrogen receptor-positive, human epidermal growth factor receptor 2 (HER2)-negative breast cancer patients.

It is of utmost importance that patients with a low risk of recurrence are identified to avoid unnecessary chemotherapy resulting in only marginal risk reduction and bearing a risk of significant toxicities.

By using a life-long Markov state transition model, we show that molecular markers such as EndoPredict ${ }^{\circledR}$ can sensibly be combined with clinical guidelines and help reduce chemotherapy usage and associated treatment costs in primary breast cancer patients.

\section{Introduction}

Predictive and prognostic markers are now routinely used to guide patient management in oncology [1]. In women with early-stage estrogen receptor-positive $\left(\mathrm{ER}^{+}\right)$, human epidermal growth factor receptor 2-negative (HER2 ${ }^{-}$) nodenegative or node-positive primary breast cancer, an individual measure of risk of distant recurrence determines whether the patient is treated with endocrine therapy alone or with additional adjuvant chemotherapy. The decision to use cytotoxic therapy weighs reduced mortality [2] against the morbidity or mortality of adverse effects [3]. Therefore, clinicians need to identify those patients with a low risk of recurrence to avoid the unnecessary use of chemotherapy and its associated toxicities for only marginal risk reduction [4].

Current clinical guidelines consider clinicopathologic factors, including tumor size, nodal status, and histological grade, to assess individual risk of recurrence. In Germany, for example, the interdisciplinary S3 guideline for the "diagnosis, therapy, and follow-up of breast cancer", which is regularly updated by experts, is used in clinical practice for the management of breast cancer [5]. Over the last few years, several molecular markers have entered the market to aid clinical decision making [6], but their long-term benefits and disadvantages for the patient and the healthcare system need to be balanced [7].

Since 2011, a new, clinically validated gene expression test (EndoPredict ${ }^{\circledR}$, Sividon Diagnostics, Köln, Germany) has been used to determine the risk of distant recurrence in patients with $\mathrm{ER}^{+}, \mathrm{HER} 2^{-}$breast cancer [8]. The EndoPredict ${ }^{\circledR}$ test is based on the assessment of the expression of eight genes of interest by quantitative real-time polymerase chain reaction (PCR) in combination with two clinical risk factors (tumor size, nodal status), which are combined to a hybrid molecular-clinicopathologic score (EPclin) [8]. EPclin adds prognostic information to other routinely measured parameters, including Ki67 [8, 9], and predicts early and late metastasis [9]. When EPclin is used in combination with established guidelines, chemotherapy use is substantially reduced without compromising patient outcome [10].

Here we evaluate the health economic impact of EPclin using a life-long Markov state transition model. We compare seven alternative strategies, three implying the use of different standard guidelines based on clinico-pathological parameters and four using EPclin, either alone or in combination with each of the three standard guidelines.

\section{Methods}

\subsection{Overview of the Breast Cancer Disease Model}

A Markov state transition model was constructed to represent the patient paths, and clinical and economic consequences, associated with each testing strategy (model implementation, see Electronic Supplementary Material S3.4). Simulated patient cohorts were entered into the model after primary surgery (Table 1). The model was comprised of three mutually exclusive health states (disease-free, metastasis, death) and had a life-long horizon (i.e., 50 cycles with a cycle length of 1 year). All patients entered the model in the disease-free state, from which point they could either remain disease free or develop distant metastases; patients with metastases could remain in this state or die (Fig. 1). Recurrence was defined as any distant metastasis event, with the assumption that $3 \%$ of metastatic patients experienced local recurrence first. Halfcycle correction was used where applicable. Effectiveness was assessed in terms of quality-adjusted life-years (QALYs) gained and life-years gained (LYG). Costs were assessed from the perspective of the German healthcare system. Non-medical direct costs and indirect costs were not taken into account in the base case, but were included in a sub-analysis. Costs are shown in 2010 euros $(€)$ and costs and effects were discounted at $3 \%$. On this basis, incremental cost-effectiveness ratios (ICERs) were 
Table 1 Characteristics of the study population

\begin{tabular}{|c|c|c|}
\hline Characteristic & $N$ & $\%$ \\
\hline Number of samples & 1,619 & 100 \\
\hline \multicolumn{3}{|l|}{ Age } \\
\hline Median (years) & 64 & \\
\hline Range (years) & $42-81$ & \\
\hline$\leq 60$ years & 551 & 44 \\
\hline$>60$ years & 1,068 & 66 \\
\hline \multicolumn{3}{|l|}{ Tumor size } \\
\hline $\mathrm{T} 1($ size $\leq 2 \mathrm{~cm})$ & 1,110 & 69 \\
\hline $\mathrm{T} 2($ size $>2 \mathrm{~cm}$ to $\leq 5 \mathrm{~cm})$ & 494 & 31 \\
\hline $\mathrm{T} 3($ size $>5 \mathrm{~cm})$ & 15 & 1 \\
\hline \multicolumn{3}{|l|}{ Nodal status } \\
\hline Negative & 1,165 & 72 \\
\hline 1-3 positive lymph nodes & 454 & 28 \\
\hline \multicolumn{3}{|l|}{ Tumor grading } \\
\hline Grade G1 & 368 & 23 \\
\hline Grade G2 & 1,196 & 74 \\
\hline Grade G3 & 53 & 3 \\
\hline \multicolumn{3}{|l|}{ Estrogen receptor ${ }^{\mathrm{a}}$} \\
\hline Low & 166 & 10 \\
\hline Medium & 521 & 32 \\
\hline High & 932 & 58 \\
\hline \multicolumn{3}{|l|}{ Progesterone receptor $^{\mathrm{a}}$} \\
\hline Negative & 335 & 21 \\
\hline Low & 277 & 17 \\
\hline Medium & 536 & 33 \\
\hline High & 471 & 29 \\
\hline \multicolumn{3}{|l|}{$\mathrm{Ki} 67^{\mathrm{b}}$} \\
\hline Low $(<14 \%)$ & 1,215 & 75 \\
\hline $\operatorname{High}(\geq 14 \%)$ & 343 & 21 \\
\hline Unknown & 61 & 4 \\
\hline \multicolumn{3}{|l|}{ Type of endocrine therapy } \\
\hline Tamoxifen & 965 & 60 \\
\hline Tamoxifen + anastrozole & 654 & 40 \\
\hline
\end{tabular}

${ }^{a}$ Classification based on the Reiner score [55]

b According to cut-off in the St. Gallen recommendations [11]

determined. Sensitivity analyses were undertaken to test the robustness of the results.

\subsection{Strategies Compared and Disease Stages}

The risk stratification strategies implemented in the model were the use of EPclin alone and the use of the German-S3 2008 guideline [5], the 2011 St. Gallen consensus recommendations [11], and the 2007 National Comprehensive Cancer Center Network (NCCN) guideline [12]. For German-S3- and St. Gallen-based stratification, patients were assigned to a low- versus a combined intermediate-/ high-risk group (referred to as "high risk" hereafter).

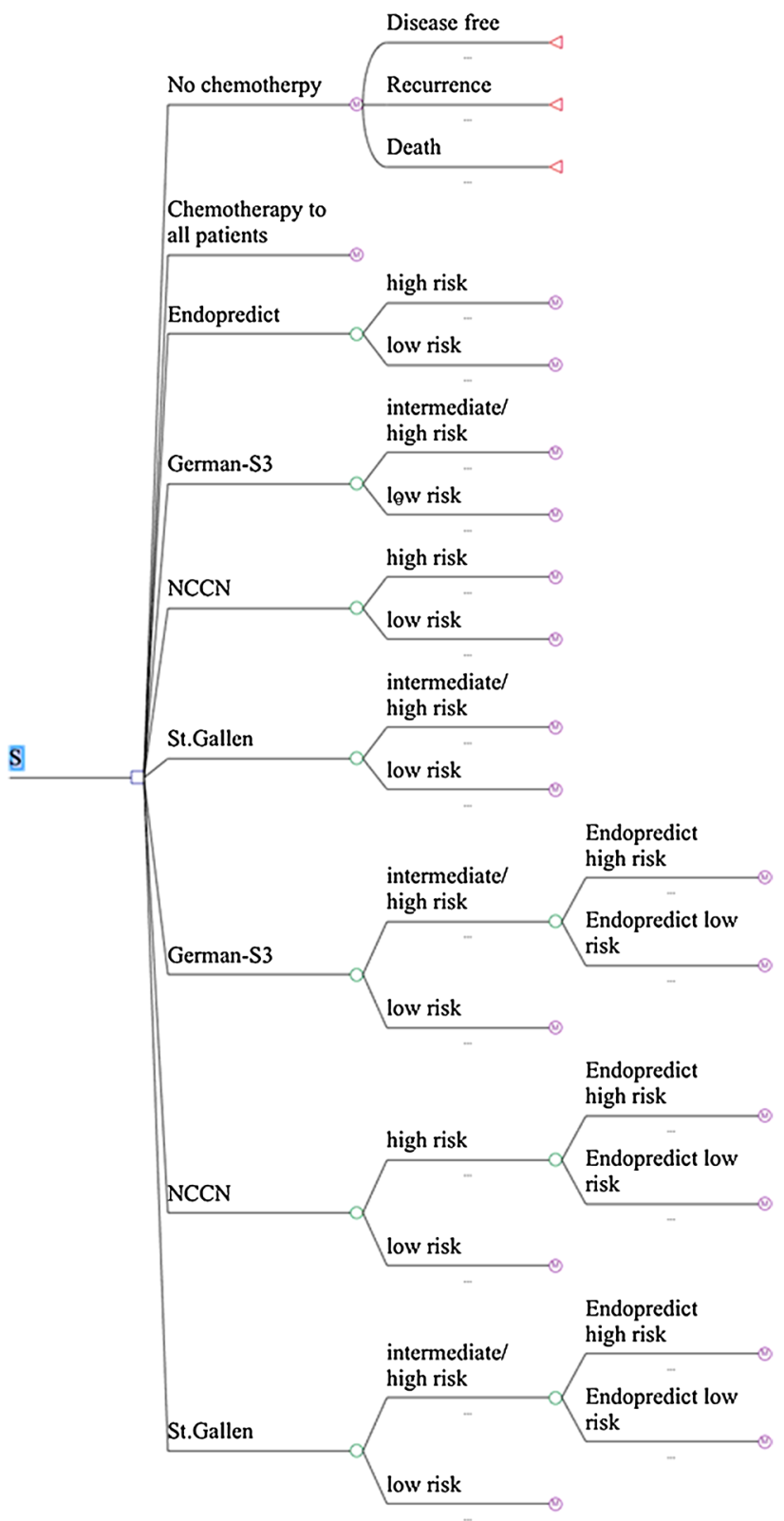

Fig. 1 Structure of Markov model. The three Markov stages are only shown for the first strategy but apply to all strategies. $M$ Markov node, $N C C N$ National Comprehensive Cancer Center Network, $S$ strategy

NCCN patients were directly classified as being at low risk or high risk since NCCN does not define an intermediate group. In addition, sequential strategies of combined guideline-based pre-stratification and subsequent testing of high-risk patients with EPclin (Fig. 1; S.1) were also included. In these strategies, only patients who were at high risk according to the respective guideline, and who had a positive molecular test, were regarded as high risk.

All patients were assigned to risk categories based on strategy-specific criteria as described above. However, in German-S3 strategies, patients with small, node-negative 
$\mathrm{T} 1 \mathrm{a} / \mathrm{b}$ grade 2 tumors were classified as low risk in contrast to the recommendations, since these patients do not usually receive chemotherapy in practice in Germany. The 2007 NCCN risk categorization was used, given that the current version is partially based on factors that were not available [e.g., results from Oncotype $\mathrm{DX}^{\circledR}$, Genomic Health, Redwood City, CA, USA]. Patients classified as being at high risk of recurrence received adjuvant chemotherapy, the remainder receiving endocrine therapy alone.

The German-S3 guideline is used in current clinical practice the Germany and was therefore chosen as the reference strategy.

\subsection{Study Population}

\subsubsection{Model Population}

We modeled a hypothetical cohort of early $\mathrm{ER}^{+}, \mathrm{HER}^{-}$ breast cancer patients with the same characteristics as seen in Austrian Breast \& Colorectal Cancer Study Group clinical trials 6 and 8 (ABCSG6/8), which were also used for the clinical validation of EndoPredict ${ }^{\circledR}[10,13,14]$. Patients were randomized to either tamoxifen for 5 years, or tamoxifen for 2 years followed by anastrozole for 3 years, without chemotherapy. Of 1,702 trial patients undergoing molecular testing, 1,619 patients were used for health economic analysis (Table 1); 83 patients with three or more positive lymph nodes were excluded since they would receive chemotherapy regardless [11]. For the St. Gallen classification, only 1,598 patients were available due to unknown Ki67 status or histologic grading.

\subsection{Clinical Model Parameters}

Low- and high-risk patient paths were modeled using transitions from the disease-free state to metastasis or death, and from metastasis to death. Parametric time-toevent modeling was used to define the transitions, and time-dependent hazards were estimated from the ABCSG6/ 8 10-year distant metastasis-free survival and overall survival (OS) data. Exponential hazard functions best fit the ABCSG data, and were hence estimated for each possible state transition. The resulting hazard rates were converted into transition probabilities for use with the Markov model (Electronic Supplementary Material S2).

The hazard functions for overall and recurrence-free survival (RFS) were calibrated using the curves seen in the ABCSG data. Since no chemotherapy was used in the ABCSG trials, the effect of adjuvant chemotherapy was derived from the largest available meta-analysis of longterm outcomes of patients treated with adjuvant chemotherapy, representing 100,000 early breast cancer patients [15]. Based on this, the relative reduction of risk of metastasis in patients receiving chemotherapy was 0.69 (standard error 0.04), regardless of their risk status.

\subsection{Utilities}

Utility scores representing health-related quality of life were derived from published sources. Utilities for breast cancer states were based on the European Quality of Life-5 Dimensions (EQ-5D) questionnaire, a widely used, selfreported, non-disease-specific, preference-based instrument $[16,17]$. A value of 0 indicates a health state equivalent to death or death itself, and 1 represents perfect health.

A comprehensive search for published literature containing information on utilities for health states of breast cancer patients was performed. The utility for the diseasefree state was 0.78 (95\% CI 0.75-0.81), based on applying the visual analog part of the EQ-5D questionnaire $[16,17]$ to 335 Swedish breast cancer patients [18], in line with other studies [18-20]. Patients receiving chemotherapy experienced a disutility of 0.07 for the first 2 years (during and after treatment) based on a literature search including 29 studies on health state utility values for early breast cancer patients [21]; 2 years was selected since several studies have indicated a long-term negative effect of chemotherapy on a patient's quality of life beyond the time assumed in our model [22-26].

The utility for patients in a metastatic state was 0.5 (95\% CI 0.39-0.61) based on the EQ-5D questionnaire (time trade-off method) filled in by clinicians [24], and was consistent with the literature (95\% CI 0.3-0.62 [21, 27, 28]).

\subsection{Medical Resource Use and Unit Costs}

The medical resource use for all patients is listed in Electronic Supplementary Material S3. Briefly, all medical interventions related to high- and low-risk patients were taken into account for disease-free, metastatic, and end-of-life therapy. The EPclin test was performed once, where applicable, prior to deciding on the use of adjuvant chemotherapy. Patients classified as high risk were treated with adjuvant chemotherapy, therapy for adverse effects, and endocrine therapy, as well as follow-up care and diagnostics (Electronic Supplementary Material S3.1). Low-risk patients received the same, except for chemotherapy, and in the reference strategies all patients either received chemotherapy or no chemotherapy. In the metastatic setting, it was assumed that all patients receive palliative care and monitoring/diagnostic interventions.

Costs are in euros and represent 2010 prices from the German healthcare perspective. Outpatient costs were based on 2010 Standard Assessment Criteria (evidence- 
based medicine (EBM)-point value 3.5048 cents) [29]. Costs for inpatients were based on the Diagnosis-Related Groups system in Germany (G-DRG) [30]. Drug acquisition costs were calculated on the basis of the standard pharmacy prices in Germany extracted from the Rote Liste 2010 [31] (Electronic Supplementary Material S3.3).

\subsection{Sensitivity Analyses}

\subsubsection{Deterministic Sensitivity Analysis}

In one-way sensitivity analyses, the precision and robustness of the results were assessed.

Variables affected by parameter uncertainty, such as prevalence of high- and low-risk scores and utility values, inclusive of the disutility associated with chemotherapy, were varied within their $95 \%$ confidence intervals, where available. OS and progression-free survival (PFS) were assessed by varying the $95 \%$ confidence intervals of the intercepts within the particular hazard function. Unit cost parameters (price of endocrine therapy or chemotherapy, price of diagnostics, costs of metastatic disease, and endof-life management) were varied by $\pm 30 \%$. Medical resource use parameters were not varied separately; it was assumed that the uncertainty present in these parameters would have been covered by the variation in unit costs.

Unit costs not subject to parameter uncertainty, such as the cost of the EndoPredict ${ }^{\circledR}$ test, were varied by $\pm 30 \%$ in scenario analyses. In another scenario it was assumed that only $70 \%$ of patients eligible for chemotherapy would actually receive it [32]. The discount rate was set to 0 and $6 \%$. A societal perspective was approximated by including indirect costs for absenteeism $(€ 5,600)$ for patients below 60 years (34\%) [33].

Hypothetical strategies representing no risk stratification or chemotherapy administration in any patient, and chemotherapy treatment of all patients regardless of their individual risk, were included in a secondary analysis to achieve a broader perspective.

\subsubsection{Probabilistic Sensitivity Analysis}

Uncertainty around the base-case results was further assessed by probabilistic sensitivity analysis (PSA; secondorder Monte-Carlo simulation), using 10,000 sets of parameter values which were randomly sampled from statistical distributions reflecting the ranges of variation used in deterministic sensitivity analysis [34]. Variables included were prevalence of high-risk groups and utility scores (beta distribution) as well as transition probabilities (i.e., intercepts of hazard functions, normal distribution) and unit costs ( $\pm 30 \%$; triangular distribution).

\section{Results}

\subsection{Base-Case Analysis}

\subsubsection{Costs}

The direct medical costs of the different strategies are shown in Table 2. The costs of chemotherapy and end-oflife management had the highest impact on total (life-long) per patient costs. The average life-long cost per patient treated according to clinical guidelines ranged from $€ 31,699$ (German-S3) to $€ 33,756$ (NCCN), with incremental costs for guideline-driven strategies between $€ 506$ (St. Gallen) and $€ 2,057$ (NCCN) in comparison with reference (German-S3). EPclin-based risk stratification in combination with guidelines reduced the average life-long cost per patient to between $€ 28,268$ (St. Gallen/EPclin) and $€ 28,987$ (NCCN/EPclin). Compared with German-S3, the combined strategies with EPclin/St. Gallen, EPclin/German-S3, or EPclin/NCCN would save $€ 3,431, € 3,388$, or $€ 2,858$ per patient over a long-term horizon, respectively. The cost of the EPclin test $(€ 1,819)$ was more than compensated for in these strategies by savings due to reduced chemotherapy use and reduced adverse effects.

\subsection{Effect}

The different testing strategies led to differential clinical outcomes in terms of QALYs gained (Table 3). Some patients inappropriately received chemotherapy or endocrine therapy alone, which resulted in a loss of QALYs (due to the disutility associated with chemotherapy treatment or foregone effect of chemotherapy, respectively). Among guideline-based strategies, German-S3 yielded the most QALYs (13.169). EPclin alone was superior to all other strategies (13.173 QALYs) in this respect.

Guidelines alone and combined strategies achieved 16.968 (St. Gallen/EPclin) to 17.018 (NCCN) LYG. Undiscounted LYG ranged from 28.108 to 28.227.

\subsection{Cost Effectiveness}

The strategies of St. Gallen/EPclin, German-S3/EPclin, NCCN/EPclin, and EPclin alone were dominant in comparison with the German-S3 reference strategy; i.e., they showed lower costs and higher QALY gains. Compared to St. Gallen alone, the combined St. Gallen/EPclin strategy would save $€ 3,937$ and gain 0.005 QALYs per patient on average, whereas German-S3/EPclin versus German-S3 alone would save $€ 3,388$ and gain 0.002 QALYs per patient. NCCN/EPclin would save $€ 4,915$ and gain 0.007 QALYs versus NCCN alone (Table 3). 
Table 2 Average per patient cost $(€)$ of breast cancer management for different testing and non-testing strategies

\begin{tabular}{|c|c|c|c|c|c|c|c|}
\hline Strategy & St. Gallen/EPclin & German-S3/EPclin & NCCN/EPclin & EPclin & German-S3 & St. Gallen & $\mathrm{NCCN}$ \\
\hline \multicolumn{8}{|c|}{ Recourses accumulated in the first year of therapy } \\
\hline EndoPredict $^{\circledR}$ & 1,451 & 1,370 & 1,708 & 1,819 & 0 & 0 & 0 \\
\hline Diagnostics & 805 & 805 & 805 & 805 & 807 & 807 & 808 \\
\hline Chemotherapy & 3,094 & 3,208 & 3,387 & 3,422 & 7,533 & 7,984 & 9,394 \\
\hline Adverse effects of chemotherapy & 399 & 414 & 437 & 441 & 971 & 1,030 & 1,211 \\
\hline \multicolumn{8}{|c|}{ Recourses accumulated in the first and subsequent years of therapy } \\
\hline Endocrine therapy & 3,284 & 3,284 & 3,284 & 3,284 & 3,286 & 3,286 & 3,286 \\
\hline Follow-up care & 4,139 & 4,139 & 4,140 & 4,140 & 4,150 & 4,150 & 4,154 \\
\hline Metastasis & 964 & 961 & 954 & 950 & 876 & 874 & 846 \\
\hline End-of-life management & 14,133 & 14,131 & 14,127 & 14,124 & 14,074 & 14,073 & 14,055 \\
\hline Total* & 28,268 & 28,311 & 28,841 & 28,987 & 31,699 & 32,205 & 33,756 \\
\hline
\end{tabular}

EPclin EndoPredict ${ }^{\circledR}$ test, NCCN National Comprehensive Cancer Center Network

* Numbers may not sum to total due to rounding

Table 3 Cost effectiveness of testing and non-testing strategies in comparison with the German-S3 guideline (reference) strategy

\begin{tabular}{llllllc}
\hline Strategy & Cost $(€)$ & $\begin{array}{l}\text { Incremental cost } \\
(€)^{\mathrm{a}}\end{array}$ & $\begin{array}{l}\text { Effects } \\
(\mathrm{QALY})\end{array}$ & $\begin{array}{l}\text { Effects } \\
(\mathrm{LYG})\end{array}$ & $\begin{array}{l}\text { Incremental effects } \\
(\mathrm{QALYs})^{\mathrm{a}}\end{array}$ & $\begin{array}{l}\text { ICER }(€ / \\
\text { QALY) }\end{array}$ \\
\hline $\begin{array}{l}\text { German S3 } \\
\text { (reference) }\end{array}$ & 31,699 & - & 13.169 & 17.006 & - & - \\
St. Gallen & 32,205 & 506 & 13.166 & 17.007 & -0.003 & Dominated \\
NCCN & 33,756 & 2,057 & 13.165 & 17.018 & -0.004 & Dominated \\
German S3/EPclin & 28,311 & $-3,388$ & 13.171 & 16.969 & 0.002 & Dominant \\
St. Gallen/EPclin & 28,268 & $-3,431$ & 13.171 & 16.968 & 0.002 & Dominant \\
NCCN/EPclin & 28,841 & $-2,858$ & 13.172 & 16.972 & 0.003 & Dominant \\
EPclin & 28,987 & $-2,712$ & 13.173 & 16.974 & 0.004 & Dominant \\
\hline
\end{tabular}

Dominated: a strategy is dominated by another if the former both costs more and is less clinically effective. Dominated strategies are excluded from the calculation of ICERs

Dominant: a strategy is dominant to the reference the former both costs less and is more effective

EPclin EndoPredict ${ }^{\circledR}$ test, ICER incremental cost-effectiveness ratio, $L Y G$ life-years gained, NCCN National Comprehensive Cancer Center Network, $Q A L Y$ quality-adjusted life-year

${ }^{a}$ In comparison with the German-S3 guideline (reference) strategy

When applying a classical rational choice approach, only non-dominated strategies would be considered and included in the ICER calculation. The use of German-S3/ EPclin would result in an ICER of $€ 208,241 /$ QALY compared with St. Gallen/EPclin, the least costly of the nondominated strategies (QALYs gained: 0.0002, additional costs: €43). The use of EPclin alone would imply an ICER of $€ 294,881 / \mathrm{QALY}$ compared with German-S3/EPclin (QALYs gained: 0.002, additional costs: €676). NCCN/ EPclin would be weakly dominated (Fig. 2a).

In a secondary analysis, two hypothetical strategies (no chemotherapy to any patient; chemotherapy to all patients) were added to put the impact of guideline- and EPclin-based decision making into perspective. The use of the St. Gallen/EPclin showed the most favorable ICER compared with the least costly strategy of no chemotherapy ( $€ 77,141 / \mathrm{Q} A L Y)$ (see Electronic Supplementary Material S5.1-5.2).

Cost effectiveness in terms of cost per LYG is shown in Fig. 2b. St. Gallen/EPclin, German-S3/EPclin, German-S3, and NCCN dominated the other strategies. Based on rational choice criteria, German-S3/EPclin had an ICER of $€ 33,617 / \mathrm{LYG}$ versus St. Gallen/EPclin.

Approximately 71,660 new breast cancer patients are registered annually in Germany (2008), of whom $55 \%$ would be eligible for a combined strategy (i.e., 39,413 patients) [35]. The German-S3/EPclin strategy would therefore lead to an annual saving of $€ 134$ million, and a gain in 78.826 QALYs per year, compared with GermanS3 alone. Compared with St. Gallen alone, the St. Gallen/ EPclin strategy would save about $€ 177$ million and gain 197,065 QALYs in Germany. 
Fig. 2 Cost-effectiveness plane: a cost per qualityadjusted life-year gained; $\mathbf{b}$ cost per life-year gained. A strategy is dominated by another if the former both costs more and is less clinically effective.

Dominated strategies are excluded from the calculation of incremental cost-effectiveness ratios. EUR euros, NCCN National Comprehensive Cancer Center Network
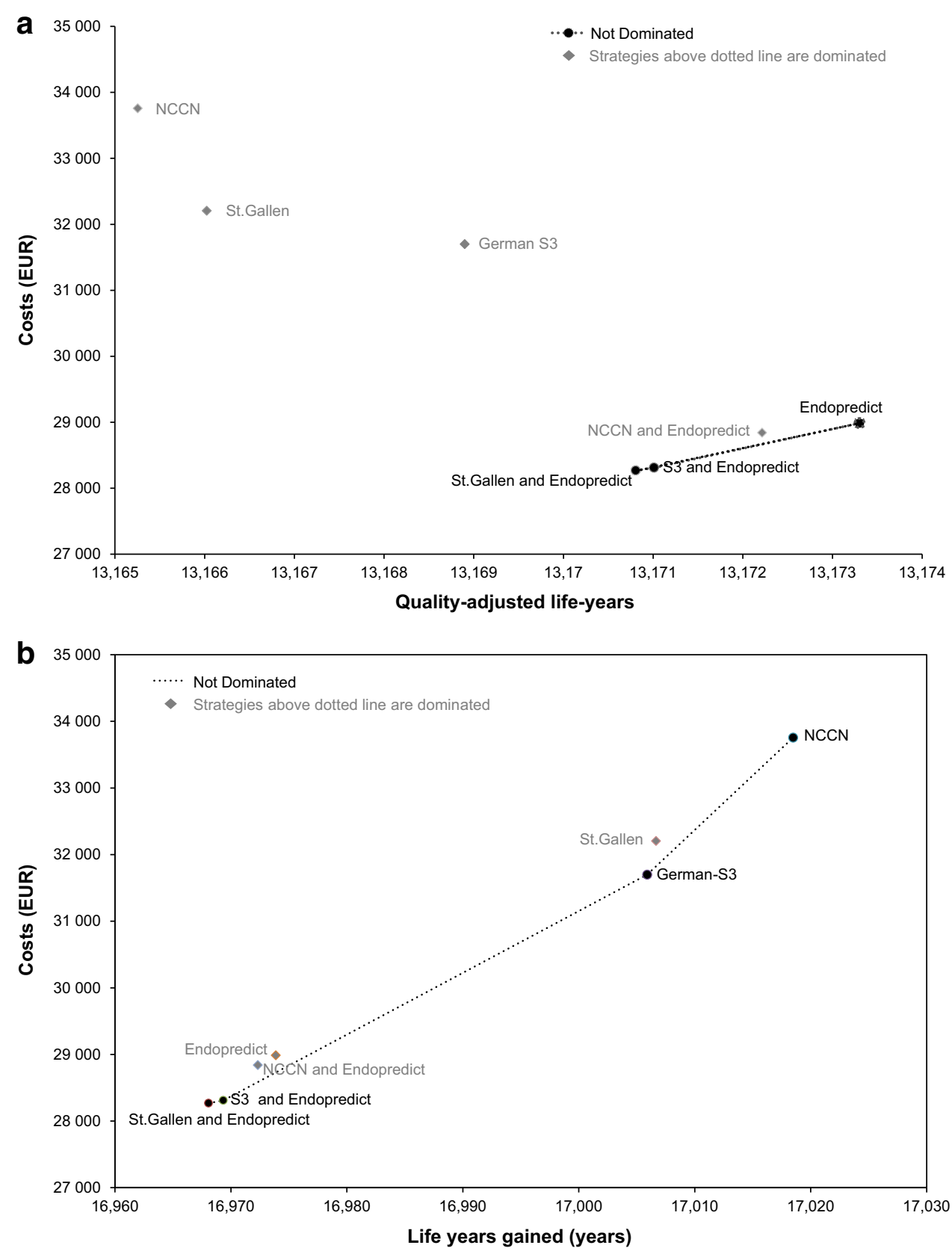

\subsection{Sensitivity Analysis}

The deterministic and scenario analyses led to a slight variation in the rank order of the combined strategies, although their advantage remained. Variation of the discount rate, the disutility due to chemotherapy, hazard rates, and the prevalence of some high-risk classifications had a strong impact on costs and effects, but cost effectiveness was essentially unaffected (Electronic Supplementary Material S4-5). Reducing chemotherapy use among eligible patients to $70 \%$ yielded reduced costs and QALYs for all strategies, and the NCCN/EPclin combination and
EPclin alone became dominated. PSA results are shown in Fig. 3 and Electronic Supplementary Material S6.

\section{Discussion}

Our study is the first health economic analysis of the EPclin test. EPclin has acceptable health economic characteristics from the perspective of the German healthcare system. Combining the St. Gallen guideline with EPclin testing of intermediate-/high-risk patients is beneficial and has the potential to aid clinical decision making on the use of 
Fig. 3 Probabilistic sensitivity analysis results: a cost versus quality-adjusted life-years; b cost per life-year gained. The cost-effectiveness acceptability curves show probabilistic sensitivity analysis-based probabilities of strategies being cost effective. For different willingness-to-pay thresholds, different strategies may be optimal. EUR euros, $L Y G$ lifeyears gained, NCCN National Comprehensive Cancer Center Network, QALYS qualityadjusted life-years
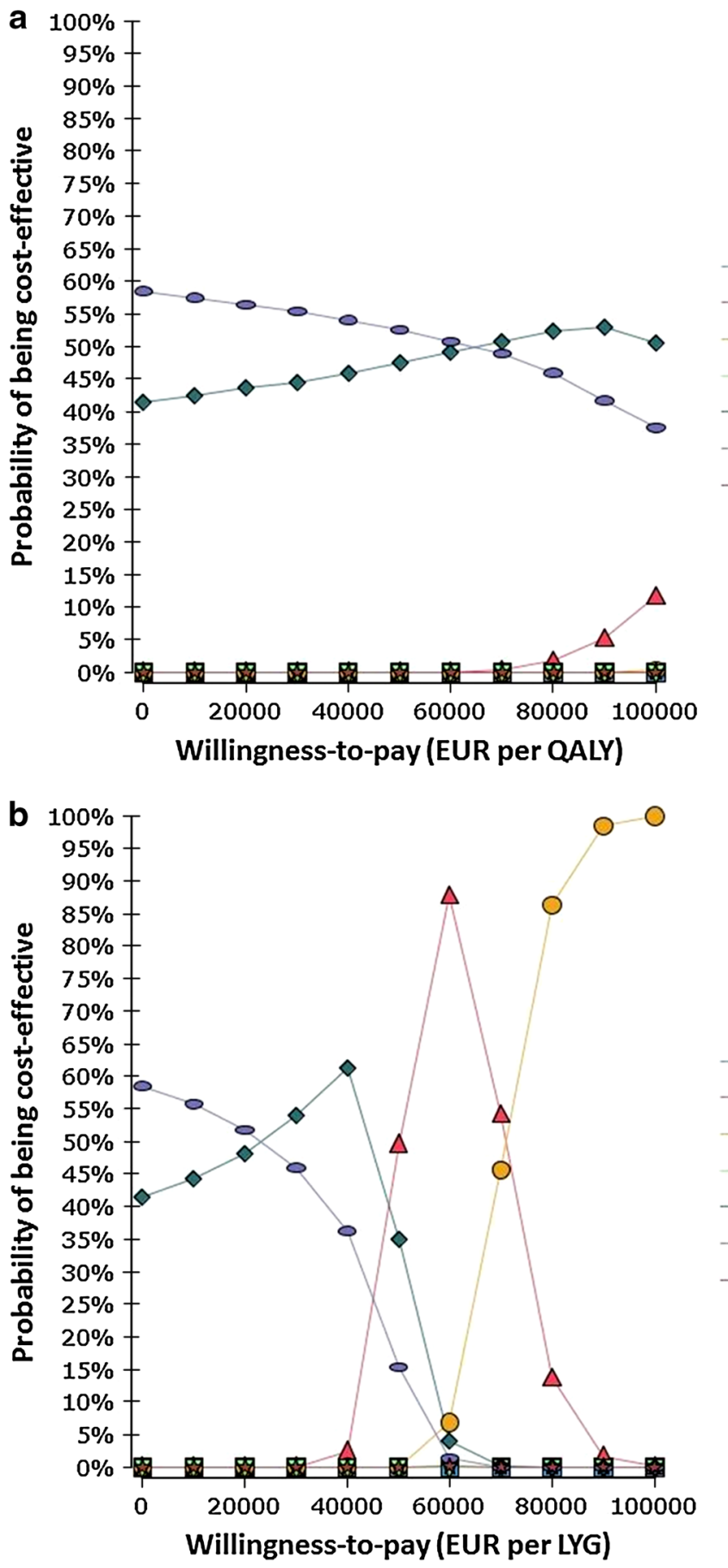

Endopredict

$\triangle$ German S3

O- NCCN

$\nabla$ NCCN and Endopr

$\diamond$ S3 and Endopredict

- St. Gallen and Endopr

it St.Gallen $\square$ Endopredict

$\triangle$ German S3

- NCCN

$\nabla$ NCCN and Endopr

$\diamond$ S3 and Endopredict

- St. Gallen and Endopr

st St.Gallen adjuvant chemotherapy in $\mathrm{ER}^{+}, \mathrm{HER}^{-}$early breast cancer patients. The robustness of this result was confirmed by extensive sensitivity analyses.

Cost-effectiveness thresholds for clinical interventions vary across countries. For example, in the USA they range from $\$ U S 50,000$ to $\$ U S 100,000$ (€36,600-73,200) per QALY gained and from $£ 20,000$ to $£ 30,000$ $(€ 23,000-35,000)$ per QALY gained in the UK, although actual resource allocations may differ [36]. In our base- case analysis, St. Gallen/EPclin, German-S3/EPclin, NCCN/EPclin, and EPclin alone were dominant strategies. Compared to current, guideline-based clinical practice, adding EPclin yielded better clinical outcomes on the QALY scale, at lower costs.

Our analysis did not take into account strategies involving other genetic testing platforms. The main reason for this was lack of information on the results the Oncotype $\mathrm{DX}^{\circledR}$ multigene test or other genetic tests would have 
yielded in the ABCSG6/8 patients, which formed the basis of this analysis.

The Oncotype DX ${ }^{\circledR}$ multigene test was recommended for use in routine practice in the USA [37] in 2007, and the health economic implications of this have been thoroughly investigated [7, 33, 38-40]. Although many studies have claimed cost effectiveness, uncertainties around their estimates were not always fully addressed [7]. Input parameters, including amount of chemotherapy use, recurrence rate, quality of life, time horizon, and the test cost seemed to most strongly influence the results [41].

Blohmer et al. [33] developed a Markov model to assess the costs and effects of using Oncotype $\mathrm{DX}^{\circledR}$ prior to adjuvant chemotherapy in Germany, and reported that an Oncotype DX ${ }^{\circledR}$-based strategy would save an average of $€ 561$ and increase QALYs by 0.06 per patient. Hornberger et al. [40] compared an Oncotype DX ${ }^{\circledR}$ strategy with the NCCN guideline and reported savings of \$US2,028 $(€ 1,669)$, and a gain of 0.086 QALYs, per patient. A direct comparison between Oncotype and EPclin on the basis of these results and ours would be difficult given different modeling approaches being used, lower test costs of EPclin, and the different proportion of patients classified as low-risk by EPclin compared with Oncotype DX ${ }^{\circledR}$. Nevertheless, all these studies showed cost savings and health gains due to the usage of genetic test results. In contrast to our EPclin-specific model, the two Oncotype DX ${ }^{\circledR}$ models generated by Blohmer et al. [33, 40] and Hornberger et al. $[33,40]$ considered a predictivity for chemotherapy benefit, i.e., the relative benefit from chemotherapy was assumed to be lower in low-risk patients than in high-risk patients. This assumption was based on a significant test for treatment interaction of the Oncotype $\mathrm{DX}^{\circledR}$ test observed in the randomized (endocrine vs. endocrine-chemotherapy treatment) NSABP (National Surgical Adjuvant Breast and Bowel Project)-B20 and the SWOG (Southwest Oncology Group)-8814 clinical trials in adjuvant breast cancer patients [42, 43]. Results from neoadjuvant studies suggested that chemotherapy sensitivity may also be enhanced in patients at high risk according to EPclin [44], but so far EPclin has not been evaluated in a randomized adjuvant clinical trial to prove that EPclin selects women who will benefit from chemotherapy treatment. In order to approximate the effect of chemotherapy, we used the largest currently available, relevant meta-analysis by Peto et al. [15]. The assumed relative risk of 0.69 for distant recurrence, in patients undergoing adjuvant chemotherapy, may therefore not be entirely correct for low-risk patients, given their low overall probability of developing a recurrence. Nevertheless, the relative benefit of adjuvant chemotherapy has been shown to be independent from standard prognostic parameters such as ER status, grading, or nodal status [15]. It is worth mentioning that in the Early Breast
Cancer Trialists' Collaborative Group (EBCTCG) metaanalysis, non-cancer-related mortality was increased due to chemotherapy (relative risk $1.2, p=0.05$ ), while overall mortality was reduced (relative risk $0.84, p<0.0001$ ) [15]. In order to remain conservative, we did not apply the increase in non-cancer-related mortality, since it was unclear whether this was a true effect or a result of competing risk. We assumed that metastases can still occur even in patients with a 10-year RFS, as shown elsewhere $[45,46]$.

Hall et al. assessed the impact of Oncotype DX ${ }^{\circledR}$ versus a chemotherapy strategy for early stage lymph node-positive breast cancer patients in the UK [7]. The testing strategy achieved a small increase in life expectancy $(0.15$ LYG) or quality-adjusted life expectancy (0.16 QALY), but also an increase in cost $(£ 860, € 1,096)$. The costs were the greatest driver of the base-case ICER ( 55,529 or $€ 6,428 / \mathrm{QALY})$. The authors clearly demonstrated the potential of molecular stratification, but also showed that there is a risk of a negative balance between costs and health benefits. Importantly, in our model only $28 \%$ of patients were node positive, and therefore had a lower baseline risk of distant metastasis. Hall et al. also included an assessment of the relative risk of death due to chronic heart failure after chemotherapy, which was not considered in our more conservative model (in order to better discriminate the effects of EPclin). Cardiac events, and especially delayed cardiac death, are uncommon (especially with docetaxel-combined chemotherapy), and cannot always be linked directly to chemotherapy [47]. This explains, in part, the reason why a greater effect was observed in terms of both QALYs and LYG in the Oncotype $\mathrm{DX}^{\circledR}$-guided strategy than in our results.

Our study has both strengths and limitations. The value of a diagnostic test or guideline depends on the ability to differentiate risk. While this is normally presented as sensitivity and specificity [48], here we did not need to explicitly include these parameters because they were indirectly taken into account in the hazard rates for metastasis extracted from the actual ABCSG trial data [48]. In the absence of chemotherapy data, we had to rely on published data for the utilities and effect of chemotherapy, which in part originated from outside Germany. The utility values had to be drawn from European sources, even though clinical treatment schedules or perception of life quality can vary between countries, evaluation methods, and severity of condition [21]. Given the fact that chemotherapy is likely to negatively influence the quality of life of treated patients, we included a disutility to account for this and as similarly used in other studies [7, 22, 23, 33].

Resource use and unit costs were abstracted from a previously published German study, which might introduce uncertainty [49]. However, the model inputs were selected 
to best match our defined patient population, and extensive sensitivity analyses were performed to take uncertainty into account. A strict follow-up schedule was assumed for all patients, which might be inappropriate, especially for lowrisk patients not receiving chemotherapy; follow-up costs might therefore be even smaller for low-risk patients. The results seemed most sensitive to the hazard rates (e.g., for disease free to metastasis) and the risk classification by various strategies. One reason for this might be the low event number in some risk groups (e.g., stratified by NCCN), which may have increased the influence of chance. In order to partially overcome this problem, we pooled the hazard rates for risk of death from general causes and the risk of death in metastatic patients, thereby improving accuracy.

cThe results presented here were derived using a cohort approach and are valid for an 'average' patient. However, specific patient subgroups might respond differently, with better or worse clinical outcomes. ABCSG6/8 were trials for post-menopausal patients with lower than average risk, favoring the adoption of a more conservative model. An additional question is whether patients actually receive their chemotherapy according to guidelines [50, 51]; it has recently been shown that chemotherapy was only given to $69 \%$ of older women with node-positive and/or ER-negative cancers [32], and although the decision to opt for chemotherapy was easy for about $60 \%$ of women, $23 \%$ found it problematic [52]. Our results may suggest that the EPclin test can mostly contribute to avoiding chemotherapy treatment in patients who would be recommended for chemotherapy according to current practice using clinical guidelines. Hence, it may be most obviously relevant for younger, fit patients due to wide use of chemotherapy in this group. However, older patients have a higher risk of experiencing adverse effects of chemotherapy and may be more reluctant to undergo treatment. In this group of older patients, the test may persuade high-risk patients to undergo chemotherapy treatment and may therefore increase survival and costs over current practice. Similarly, use of the genetic test might change decisions on chemotherapy use in lymph node-negative or lymph node-positive (1-3+) patients, with both clinical and economic implications. Given the lack of information on resource use, chemotherapy effect and other parameters across these subgroups, it was not possible to analyze the cost and effects for specific patient groups. The fact that some patients may not get chemotherapy despite guideline recommendations was addressed in a scenario analysis, where only a proportion of patients were given chemotherapy. In this analysis, the costs and effects of all strategies decreased and the combined St. Gallen/EPclin and the EPclin alone strategies remained dominant. The preferences of cancer patients always need to be considered, especially when the benefits from therapies are disputable.

\section{Conclusions}

Biologic and clinical markers can provide prognostic and predictive information and determine the residual risk after standard hormone therapy and the relative risk reduction for additional chemotherapy [53, 54], sparing exposure to hazardous and expensive adverse events [3]. Our results add to the rationale for addressing this approach in terms of the health economics of clinically validated tests and guidelines, to find those patients who benefit most from chemotherapy. The present study suggests that molecular tests such as EPclin can be sensibly combined with clinical guidelines and can help reduce chemotherapy use in $\mathrm{ER}^{+}$ breast cancer patients with few other clinical risk factors [10]. Improved risk stratification can be translated into increased quality-adjusted life and is economically viable.

Acknowledgments The research leading to these results has received funding from the European Community's Seventh Framework Programme (FP7/2007-2013) under grant agreement no. 278659 and from Sividon Diagnostics GmbH, Cologne, Germany.

Financial support and conflicts of interest Financial support, including the source and number of grants, and potential conflicts of interest, for each author are as follows:

P.R. Blank, F. Gutzwiller, S. Loibl, T.D. Szucs, J.C. Brase, K.E. Weber, R. Kronenwett, and M. Schwenkglenks receive funding from the European Commission 7th Framework Programme, Call FP7HEALTH-2011-two-stage, under Grant agreement no. 278659 (RESPONSIFY).

P.R. Blank, F. Gutzwiller, T.D. Szucs, and M. Schwenkglenks have received research funding from Sividon Diagnostics $\mathrm{GmbH}$.

P. Dubsky and M. Filipits have received honoraria from Sividon Diagnostics GmbH for scientific lectures related to EndoPredict ${ }^{\circledR}$.

J.C. Brase, K.E. Weber, and R. Kronenwett are employees of Sividon Diagnostics GmbH. K.E. Weber and R. Kronenwett are shareholders of Sividon Diagnostics $\mathrm{GmbH}$.

M. Filipits, P. Dubsky, M.P. Lux, M. Rudas, R. Greil, and M. Gnant have no conflicts of interest and did not receive any funding.

Author contribution PRB and MS initiated and led the project, methodological and parameterization decisions, designed the structure of the economic aspects of the model, reviewed the literature, and drafted the manuscript. JCB, KEW, RK, and SL were involved in model design and assisted with parameterization, reviewed the literature, and reviewed and modified the manuscript.

FG, MPL, and TDS assisted with parameterization, reviewed the literature, and reviewed and modified the manuscript. MF, PD, MR, RG, and MG assisted with parameterization, contributed to data acquisition, reviewed the literature, and reviewed and modified the manuscript. All authors approved the final manuscript. PRB and MS act as the guarantors for the project.

Open Access This article is distributed under the terms of the Creative Commons Attribution Noncommercial License which 
permits any noncommercial use, distribution, and reproduction in any medium, provided the original author(s) and the source are credited.

\section{References}

1. Schilsky RL. Personalizing cancer care: American Society of Clinical Oncology presidential address 2009. J Clin Oncol. 2009;27(23):3725-30.

2. Berry DA, Cronin KA, Plevritis SK, Fryback DG, Clarke L, Zelen $\mathrm{M}$, et al. Effect of screening and adjuvant therapy on mortality from breast cancer. N Engl J Med. 2005;353(17): 1784-92.

3. Sparano JA, Wang M, Martino S, Jones V, Perez EA, Saphner T, et al. Weekly paclitaxel in the adjuvant treatment of breast cancer. N Engl J Med. 2008;358(16):1663-71.

4. Hayes DF. Targeting adjuvant chemotherapy: a good idea that needs to be proven! J Clin Oncol. 2012;30(12):1264-7.

5. Wockel A, Kreienberg R. First revision of the German S3 guideline 'diagnosis, therapy, and follow-up of breast cancer'. Breast Care (Basel). 2008;3(2):82-6.

6. Weigelt B, Reis-Filho JS, Swanton C. Genomic analyses to select patients for adjuvant chemotherapy: trials and tribulations. Ann Oncol. 2012;23(Suppl 10):x211-8.

7. Hall PS, McCabe C, Stein RC, Cameron D. Economic evaluation of genomic test-directed chemotherapy for early-stage lymph node-positive breast cancer. J Natl Cancer Inst. 2012;104(1): 56-66.

8. Filipits M, Rudas M, Jakesz R, Dubsky P, Fitzal F, Singer CF, et al. A new molecular predictor of distant recurrence in ERpositive, HER2-negative breast cancer adds independent information to conventional clinical risk factors. Clin Cancer Res. 2011;17(18):6012-20.

9. Dubsky P, Brase JC, Jakesz R, Rudas M, Singer CF, Greil R, et al.; Austrian Breast and Colorectal Cancer Study Group (ABCSG). The EndoPredict score provides prognostic information on late distant metastases in ER+/HER2- breast cancer patients. Br J Cancer. 2013;109(12):2959-64.

10. Dubsky P, Filipits M, Jakesz R, Rudas M, Singer CF, Greil R, et al. Austrian Breast and Colorectal Cancer Study Group (ABCSG). EndoPredict improves the prognostic classification derived from common clinical guidelines in ER-positive, HER2-negative early breast cancer. Ann Oncol. 2013;24(3):640-7.

11. Goldhirsch A, Wood WC, Coates AS, Gelber RD, Thurlimann B, Senn HJ. Strategies for subtypes-dealing with the diversity of breast cancer: highlights of the St. Gallen International Expert Consensus on the Primary Therapy of Early Breast Cancer 2011. Ann Oncol. 2011;22(8):1736-47.

12. American Cancer Society (ACS) and National Comprehensive Cancer Network (NCCN). Breast cancer: treatment guidelines for patients 2007. Fort Washington: National Comprehensive Cancer Network; 2007.

13. Jakesz R, Jonat W, Gnant M, Mittlboeck M, Greil R, Tausch C, et al. Switching of postmenopausal women with endocrineresponsive early breast cancer to anastrozole after 2 years' adjuvant tamoxifen: combined results of ABCSG trial 8 and ARNO 95 trial. Lancet. 2005;366(9484):455-62.

14. Schmid M, Jakesz R, Samonigg H, Kubista E, Gnant M, Menzel $\mathrm{C}$, et al. Randomized trial of tamoxifen versus tamoxifen plus aminoglutethimide as adjuvant treatment in postmenopausal breast cancer patients with hormone receptor-positive disease: Austrian breast and colorectal cancer study group trial 6. J Clin Oncol. 2003;21(6):984-90.
15. Peto R, Davies C, Godwin J, Gray R, Pan HC, Clarke M, et al. Comparisons between different polychemotherapy regimens for early breast cancer: meta-analyses of long-term outcome among 100,000 women in 123 randomised trials. Lancet. 2012; 379(9814):432-44.

16. Brooks R. EuroQol: the current state of play. Health Policy. 1996;37(1):53-72.

17. EuroQol Group. EuroQol. http://www.euroqol.org/. Accesssed Nov 2013.

18. Lidgren M, Wilking N, Jonsson B, Rehnberg C. Health related quality of life in different states of breast cancer. Qual Life Res. 2007;16(6):1073-81.

19. Zendejas B, Moriarty JP, O'Byrne J, Degnim AC, Farley DR, Boughey JC. Cost-effectiveness of contralateral prophylactic mastectomy versus routine surveillance in patients with unilateral breast cancer. J Clin Oncol. 2011;29(22):2993-3000.

20. Conner-Spady BL, Cumming C, Nabholtz JM, Jacobs P, Stewart D. A longitudinal prospective study of health-related quality of life in breast cancer patients following high-dose chemotherapy with autologous blood stem cell transplantation. Bone Marrow Transpl. 2005;36(3):251-9.

21. Peasgood T, Ward SE, Brazier J. Health-state utility values in breast cancer. Expert Rev Pharmacoecon Outcomes Res. 2010;10(5): 553-66.

22. Rey D, Bouhnik AD, Mancini J, Bendiane MK, Séror V, Viens P. Self-reported cognitive impairment after breast cancer treatment in young women from the ELIPPSE40 cohort: the long-term impact of chemotherapy. Breast J. 2012;18(5):406-14.

23. Whyte S, Cooper KL, Stevenson MD, Madan J, Akehurst R. Cost-effectiveness of granulocyte colony-stimulating factor prophylaxis for febrile neutropenia in breast cancer in the United Kingdom. Value Health. 2011;14(4):465-74.

24. Hind D, Ward S, De Nigris E, Simpson E, Carroll C, Wyld L. Hormonal therapies for early breast cancer: systematic review and economic evaluation. Health Technol Assess. 2007;11(26): 3-4, 9-11, 1-134.

25. Yamada TH, Denburg NL, Beglinger LJ, Schultz SK. Neuropsychological outcomes of older breast cancer survivors: cognitive features ten or more years after chemotherapy. J Neuropsychiatry Clin Neurosci. 2010;22(1):48-54.

26. Koppelmans V, Breteler MM, Boogerd W, Seynaeve C, Gundy C, Schagen SB. Neuropsychological performance in survivors of breast cancer more than 20 years after adjuvant chemotherapy. J Clin Oncol. 2012;30(10):1080-6.

27. Tengs TO, Wallace A. One thousand health-related quality-of-life estimates. Med Care. 2000;38(6):583-637.

28. Younis T, Rayson D, Skedgel C. The cost-utility of adjuvant chemotherapy using docetaxel and cyclophosphamide compared with doxorubicin and cyclophosphamide in breast cancer. Curr Oncol. 2011;18(6):e288-96.

29. Bayerische Euro-Gebührenordnung und EBM-Änderungen ab 1. Januar und 1. April 2010. http://www.kvb.de. Accessed Nov 2013.

30. Institut für das Entgeltsystem im Krankenhaus: German DRG. 2010. http://www.g-drg.de/cms/. Accessed Nov 2013.

31. Rote Liste. http://www.rote-liste.de/. Accessed Nov 2013.

32. Mandelblatt JS, Sheppard VB, Hurria A, Kimmick G, Isaacs C, Taylor KL, et al. Breast cancer adjuvant chemotherapy decisions in older women: the role of patient preference and interactions with physicians. J Clin Oncol. 2012;28(19):3146-53.

33. Blohmer JU, Rezai M, Kummel S, Kuhn T, Warm M, Friedrichs $\mathrm{K}$, et al. Using the 21-gene assay to guide adjuvant chemotherapy decision-making in early-stage breast cancer: a cost-effectiveness evaluation in the German setting. J Med Econ. 2013;16(1):30-40.

34. Briggs AH, Goeree R, Blackhouse G, O'Brien BJ. Probabilistic analysis of cost-effectiveness models: choosing between 
treatment strategies for gastroesophageal reflux disease. Med Decis Making. 2002;22(4):290-308.

35. Krebs in Deutschland 2007/2008. Häufigkeiten und Trends. Berlin: Robert Koch-Institut (Hrsg.); 2012.

36. Braithwaite RS, Meltzer DO, King JT Jr, Leslie D, Roberts MS. What does the value of modern medicine say about the $\$ 50,000$ per quality-adjusted life-year decision rule? Med Care. 2008;46(4):349-56.

37. Harris L, Fritsche H, Mennel R, Norton L, Ravdin P, Taube S, et al. American Society of Clinical Oncology 2007 update of recommendations for the use of tumor markers in breast cancer. J Clin Oncol. 2007;25(33):5287-312.

38. Jahn B, Rochau U, Kurzthaler C, Arvandi M, Saverno K, et al. Evaluation of breast cancer test-treatment strategies using decision-analytic modeling-preliminary results for the 21-gene assay recurrence score. IMPAKT 2013; 2-4 May 2013; Brussels.

39. Tsoi DT, Inoue M, Kelly CM, Verma S, Pritchard KI. Costeffectiveness analysis of recurrence score-guided treatment using a 21-gene assay in early breast cancer. Oncologist. 2012;15(5): 457-65.

40. Hornberger J, Cosler LE, Lyman GH. Economic analysis of targeting chemotherapy using a 21-gene RT-PCR assay in lymphnode-negative, estrogen-receptor-positive, early-stage breast cancer. Am J Manag Care. 2005;11(5):313-24.

41. Bartlett J, Canney P, Campbell A, Cameron D, Donovan J, Dunn $J$, et al. Selecting breast cancer patients for chemotherapy: the opening of the UK OPTIMA trial. Clin Oncol (R Coll Radiol). 2013;25(2):109-16.

42. Albain KS, Barlow WE, Shak S, Hortobagyi GN, Livingston RB, Yeh IT, Breast Cancer Intergroup of North America, et al. Prognostic and predictive value of the 21-gene recurrence score assay in postmenopausal women with node-positive, oestrogenreceptor-positive breast cancer on chemotherapy: a retrospective analysis of a randomised trial. Lancet Oncol. 2010;11(1):55-65.

43. Paik S, Tang G, Shak S, Kim C, Baker J, Kim W, et al. Gene expression and benefit of chemotherapy in women with nodenegative, estrogen receptor-positive breast cancer. J Clin Oncol. 2006;24(23):3726-34.

44. Brase JC, MC Gehrmann, Petry C, Weber KE, Schmidt M, Kölbl $\mathrm{H}$, et al. The EndoPredict score is a response predictor for neoadjuvant chemotherapy in ER-positive, HER2-negative breast cancer [poster no. P1-06-26]. Cancer Res. 2011;71(24 Suppl 3):P1-06-26.

45. Esserman LJ, Moore DH, Tsing PJ, Chu PW, Yau C, Ozanne E, et al. Biologic markers determine both the risk and the timing of recurrence in breast cancer. Breast Cancer Res Treat. 2011;129(2):

607-16.

46. Jatoi I, Anderson WF, Jeong JH, Redmond CK. Breast cancer adjuvant therapy: time to consider its time-dependent effects. J Clin Oncol. 2011;29(17):2301-4.

47. Roche H, Fumoleau P, Spielmann M, Canon JL, Delozier T, Serin D, et al. Sequential adjuvant epirubicin-based and docetaxel chemotherapy for node-positive breast cancer patients: the FNCLCC PACS 01 trial. J Clin Oncol. 2006;24(36):5664-71.

48. Sanghera S, Orlando R, Roberts T. Economic evaluations and diagnostic testing: an illustrative case study approach. Int $\mathrm{J}$ Technol Assess Health Care. 2013;29(1):53-60.

49. Lux MP, Reichelt C, Karnon J, Tanzer TD, Radosavac D, Fasching PA, et al. Cost-benefit analysis of endocrine therapy in the adjuvant setting for postmenopausal patients with hormone receptor-positive breast cancer, based on survival data and future prices for generic drugs in the context of the German health care system. Breast Care (Basel). 2011;6(5):381-9.

50. Harlan LC, Abrams J, Warren JL, Clegg L, Stevens J, BallardBarbash R. Adjuvant therapy for breast cancer: practice patterns of community physicians. J Clin Oncol. 2002;20(7):1809-17.

51. Palazzi M, De Tomasi D, D'Affronto C, Richetti A, Valli MC, Meregalli S, et al. Are international guidelines for the prescription of adjuvant treatment for early breast cancer followed in clinical practice? Results of a population-based study on 1,547 patients. Tumori. 2002;88(6):503-6.

52. Shelton RC, Hillyer GC, Hershman DL, Leoce N, Bovbjerg DH, Mandelblatt JS, et al. Interpersonal influences and attitudes about adjuvant therapy treatment decisions among non-metastatic breast cancer patients: an examination of differences by age and race/ethnicity in the BQUAL study. Breast Cancer Res Treat. 2012;137(3):817-28.

53. Kiberstis P, Marshall E. Cancer crusade at 40. Celebrating an anniversary. Introduction. Science. 2011;331(6024):1539.

54. Tang G, Cuzick J, Costantino JP, Dowsett M, Forbes JF, Crager $\mathrm{M}$, et al. Risk of recurrence and chemotherapy benefit for patients with node-negative, estrogen receptor-positive breast cancer: recurrence score alone and integrated with pathologic and clinical factors. J Clin Oncol. 2012;29(33):4365-72.

55. Regitnig P, Reiner A, Dinges HP, Hofler G, Muller-Holzner E, Lax SF, et al. Quality assurance for detection of estrogen and progesterone receptors by immunohistochemistry in Austrian pathology laboratories. Virchows Arch. 2002;441(4):328-34. 